\title{
3-D TIGER Observations of Radio Galaxies
}

\author{
G. Adam, ${ }^{1}$ B. Rocca-Volmerange ${ }^{2}$ P. Ferruit, ${ }^{1}$ and R. Bacon ${ }^{1}$ \\ ${ }^{1}$ CRAL, Observatoire de Lyon, F-69561 St. Genis Laval Cedex, France \\ ${ }^{2}$ IAP, CNRS, 98 bis Bd. Arago, F-75014 Paris, France
}

\section{Cosmological Stellar-Population Evolution Study}

The cosmological evolution of stellar populations can be studied by observing, e.g, high- $z$ radio galaxies, and comparing their SEDs to those of local objects. But in such objects, the nuclear activity strongly influences the optical properties; to disentangle the superposition of spatial and spectral effects, complete 3-D data cubes are needed, such as those that can be obtained with the pioneering 3-D instrument TIGER.

\section{The Ly $\alpha$ Morphology of $4 \mathrm{C} 41.17$}

4C $41.17(z=3.8)$ was observed at CFHT with good seeing $\left(0^{\prime \prime} .5\right)$ for a total integration time of only $1.86 \mathrm{~h}$, with $0^{\prime \prime} .6$ and $8 \AA$ sampling over a $12^{\prime \prime}$ field. A main Ly $\alpha$ peak shows a brightness of $5.9 \times 10^{-8} \mathrm{ergs} \mathrm{cm}^{-2} \mathrm{arcsec}^{-2}$; over $2.7 \times 5.4 \mathrm{kpc}^{2}\left(H_{0}=75 \mathrm{~km} \mathrm{~s}^{-1} \mathrm{Mpc}^{-1}, q_{0}=0.5\right)$, it emits $4.8 \times 10^{43} \mathrm{ergs} \mathrm{s}^{-1}$. A secondary peak is seen to the west, and both peaks are on the radio axis defined by the two strong $15 \mathrm{GHz}$ components $\mathrm{B} 2$ and $\mathrm{B} 3$, the flat-spectrum $\mathrm{N}$ component and the high-resolution $H S T$ components $\mathrm{H} 2, \mathrm{H} 3$, and $\mathrm{H} 4$. General curvature of the Ly $\alpha$ axis is noticeable, extending the known radio-axis twist. Filaments protrude out of the central region; their very inner parts are seen on Ly $\alpha$ HST archive images.

A detailed analysis will be presented elsewhere, but some salient features are already clear. The dense central part has a size of $35 \times 18 \mathrm{kpc}^{2}$; if a merger is underway, the bulk of the galaxy seems to be already formed at $z=3.8$. The curved radio jet follows the Ly $\alpha$ isophotes over at least $18 \mathrm{kpc}$; this would favor a star-formation process rather than scattering to explain the alignment effect. It is difficult to invoke a jet/IGM turbulent interaction at a cocoon frontier to explain the high velocity dispersions (up to $2000 \mathrm{~km} \mathrm{~s}^{-1}$ ), as no emission enhancement is seen where the frontal bow shocks are expected. The galaxy stellar continuum and the depression on the blue side of Ly $\alpha$ are detected by integration over several spatial elements; study of the SED is in progress. The dip between the two Ly $\alpha$ peaks may trace an intervening absorber, or a large dust component acting as an efficient Ly $\alpha$-killer, possibly the host disk plane masking the compact core N. Finally, the crowded Ly $\alpha$ neighborhood of galaxy and the observed velocities may trace the last stages of a merger. 

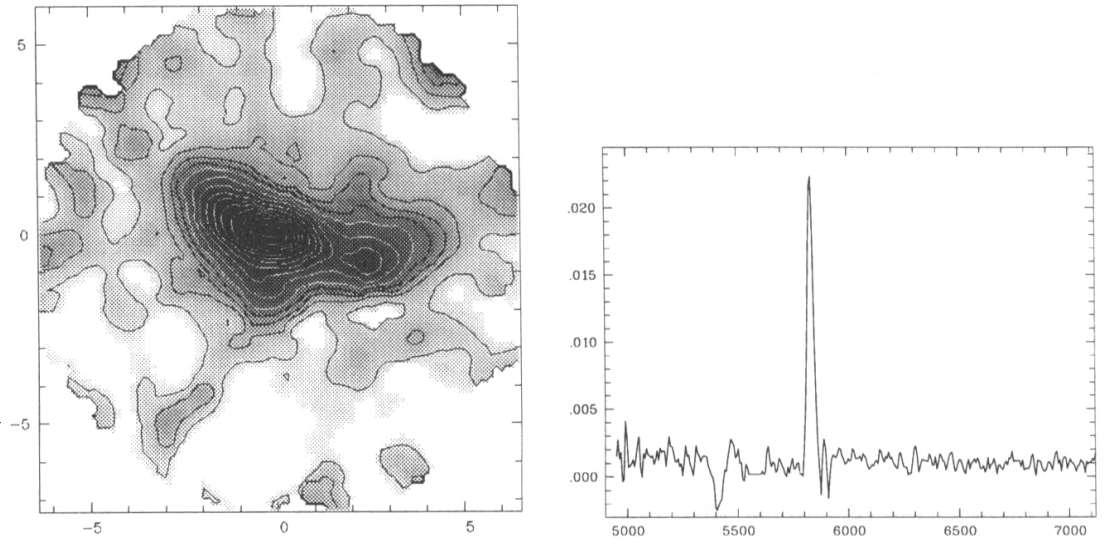

Figure 1. Left: 4C 41.17 Ly $\alpha$ image, continuum subtracted, reconstructed from individual spectra and smoothed with a $0^{\prime \prime} .8$ (FWHM) Gaussian filter; coordinates are arcseconds. Right: spectrum of the central zone, integrated over a square region $14^{\prime \prime} .7$ on a side.

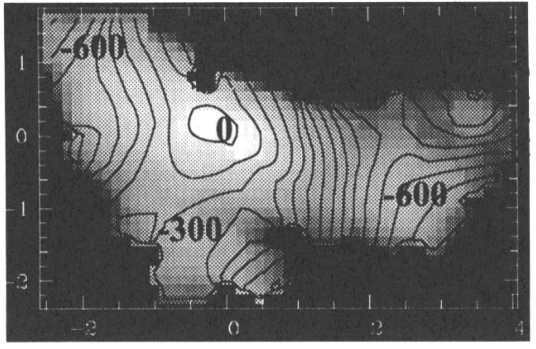

Velocities $(\mathrm{km} / \mathrm{s})$

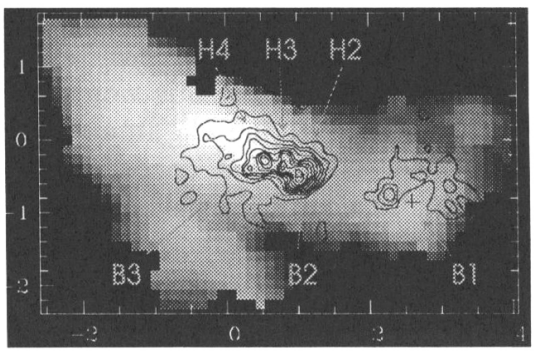

HST continuum morphology

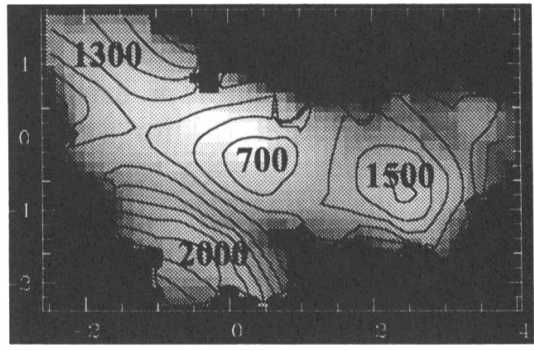

Velocities dispersion $(\mathrm{km} / \mathrm{s})$

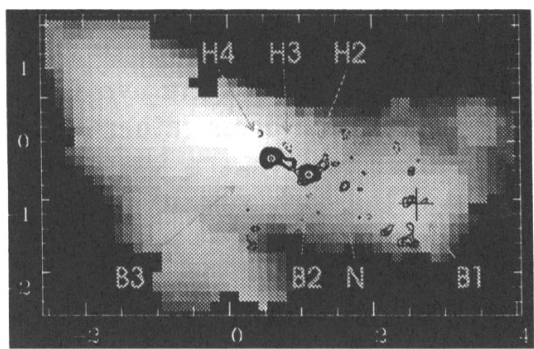

15 Ghz VLA image, resolution $0.15^{\prime \prime}$

Figure 2. 4C 41.17 velocities, velocity dispersions, HST continuum, and $15 \mathrm{GHz}$ radio maps, superposed on a Ly $\alpha$ image; coordinates are arcseconds. 